01

\title{
Локализация возбуждений вблизи тонкого дефектного слоя с нелинейными свойствами, разделяющего линейный и нелинейный кристаллы
}

\author{
(C) C.E. Савотченко
}

Белгородский государственный технологический университет им. В.Г. Шухова, 308012 Белгород, Россия

e-mail: savotchenkose@mail.ru

Поступило в Редакцию 27 октября 2017 г.

В окончательной редакции 27 октября 2017 г.

Принято к публикации 11 марта 2019 г.

\begin{abstract}
Показано, что вблизи тонкого дефектного слоя с нелинейными свойствами, отделяющего линейную среду от нелинейной среды керровского типа, существуют локализованные и квазилокальные стационарные состояния. Локализованные состояния характеризуются монотонно убывающей амплитудой поля по обе стороны от границы раздела сред. Квазилокальные состояния описываются полем в виде стоячей волны в линейной среде и монотонно убывающим полем в нелинейной среде. Проанализированы контакты с нелинейными самофокусирующими и дефокусирующими средами. Математическая формулировка предложенной модели представляет собой систему линейного и нелинейного уравнений Шредингера с нелинейным относительно поля потенциалом, моделирующим тонкий дефектный слой. Получены дисперсионные соотношения, определяющие энергию локальных и квазилокальных состояний. В явном аналитическом виде выражения для энергий получены в предельных случаях и указаны условия их существования.
\end{abstract}

Ключевые слова: нелинейное уравнение Шредингера, граница раздела сред, плоский дефект, солитоны, локализованные состояния.

DOI: $10.21883 /$ JTF.2019.09.48054.2560

\section{Введение}

Большую роль в проектировании нелинейных волноводных устройств играет изучение особенностей управляемой локализации электромагнитных волн. Распространяемые вдоль волноводов возбуждения электромагнитного поля, которые быстро затухают при удалении от границы раздела сред, принято называть нелинейными поверхностными волнами. Их теоретическое исследование $[1,2]$ и экспериментальное наблюдение [3] началось достаточно давно. Тем не менее изучение нелинейных поверхностных волн, распространяющихся вдоль границ раздела диэлектрических сред с различными характеристиками, остается актуальным в связи с их широким применением в оптических системах хранения данных [4].

Большинство теоретических работ посвящено получению распределения полей и дисперсионных соотношений для волн, распространяющихся вдоль границ раздела линейной и нелинейной сред $[5,6]$ и трехслойных структур [7]. Модификации моделей границ раздела нелинейных кристаллов рассматривались в работах $[8,9]$. Особенности локализации нелинейных волн на границе раздела нелинейных сред с пространственной дисперсией анализировались в [10]. Взаимодействие вблизи дефекта связанных солитонных состояний, относящихся к различным состояниям двухуровневой системы, рассмотрено в [11]. Учет внутренней структуры тонкой про- слойки между нелинейными кристаллами был проведен в [12].

Расчет полей нелинейных волн, как правило, проводится на основе нелинейного уравнения Шредингера (НУШ), которое для случая среды с эффектом Керра содержит кубическое (относительно искомого поля) слагаемое [13]. Следует отметить, что НУШ широко применятся для аналитического описания эффектов локализации полей различной физической природы, в том числе магнитного [14] и упругого [15].

Теоретическое изучение взаимодействия нелинейных возбуждений с плоским или точечным дефектом проводится с использованием математических моделей на основе НУШ, потенциал в котором описывает дефект. Во многих случаях задача сводится к одномерной, и тогда для моделирования дефекта применяется короткодействующий потенциал в виде

$$
U(x)=U_{0} \delta(x) .
$$

где $\delta(x)$ - дельта-функция Дирака, $U_{0}$ - интенсивность взаимодействия возбуждения с дефектом, расположенным в начале координат (иногда данная величина называется „мощностью“ дефекта). При $U_{0}>0$ возбуждение отталкивается от дефекта, а при $U_{0}<0-$ притягивается.

Ясно, что короткодействующий потенциал вида (1) не может в достаточной степени описать влияние свойств тонкого дефектного слоя на эффекты локализации возбуждений. С целью изучения возможностей управления 
распространением волн в слоистых структурах через границы раздела сред, учитывающие их внутренние нелинейные свойства, было предложено использовать потенциал с квадратичной нелинейностью относительно искомого поля [16-18]. В случае слабой связи между плоскопараллельными волноводами амплитуда поля в них намного больше средней амплитуды поля во всем кристалле, поэтому нелинейные слагаемые было предложено учитывать только внутри самих волноводов. Анализ особенностей распространения локализованных возбуждений в системах с такими свойствами является актуальным в связи с широким применением слоистых структур, содержащих плоскопараллельные волноводы, в нелинейной оптике.

В настоящей работе предложено обобщение представленной в [16-18] модели тонкого дефектного слоя, который внутри характеризуется керровской нелинейностью, на случай контакта линейной среды с кристаллом с керровской нелинейностью. Будет проведено исследование локализации возбуждений вблизи одного нелинейного тонкого дефектного слоя, обладающего волноводными свойствами, и показано, что существует несколько типов стационарных состояний, имеющих локализованные составляющие. Будут рассмотрены случаи контакта линейной среды с нелинейными средами керровского типа с различными знаками нелинейности. Основной целью настоящей работы является нахождение энергии локализованных состояний всех видов, возникающих в рассматриваемой системе, и определение условий их существования.

Следует отметить, что в работе [7] были рассмотрены стационарные нелинейные поверхностные волны вблизи границы раздела сред с различными характеристиками, в том числе и контакта линейной и нелинейной сред, но без учета особенности структуры границы раздела и ее влияния на локализацию волн. С другой стороны, в [16,17] были проанализированы особенности локализации возбуждений вблизи границы раздела с нелинейными свойствами между нелинейными средами, но с одинаковыми характеристиками. Отличительной особенностью настоящей работы является то, что в ней проведено исследование локализации возбуждений вблизи границы раздела с нелинейными свойствами между средами с различными характеристиками, в частности, между линейной средой и средой кубической нелинейностью.

\section{1. Уравнения модели}

Будем считать, что тонкая прослойка, разделяющая кристаллы с гармоническим (линейная среда) и анграмоническим (нелинейная среда) взаимодействием элементарных возбуждений, расположена в плоскости $y O z$ перпендикулярно оси $O x$. Толщина прослойки существенно меньше характерного расстояния локализации возбуждений.
Линейный (гармонический) кристалл занимает полупространство $x<0$, а нелинейный (ангармонический) кристалл $-x>0$. Тогда параметр нелинейности в НУШ будет иметь вид

$$
\gamma(x)= \begin{cases}0, & x<0 \\ \gamma, & x>0\end{cases}
$$

Граница раздела, как плоский дефект, создает возмущение характеристик среды, сосредоточенных на расстояниях, существенно меньших ширины локализации рассматриваемых возбуждений.

Следуя $[17,18]$, для описания плоского дефекта с нелинейными свойствами будем использовать потенциал в виде

$$
U(x)=U_{0} \delta(x)|\psi|^{2} .
$$

Будем рассматривать взаимодействие нелинейных возбуждений, локализованных вблизи тонкого слоя с нелинейными свойствами, на основе одномерного НУШ (принято $\hbar=1)$ :

$$
i \psi_{t}^{\prime}=-\frac{1}{2 m} \psi_{x x}^{\prime \prime}+\Omega(x) \psi-\gamma(x)|\psi|^{2} \psi+U(x) \psi,
$$

где $m$ - эффективная масса возбуждения,

$$
\Omega(x)= \begin{cases}\Omega_{1}, & x<0, \\ \Omega_{2}, & x>0,\end{cases}
$$

где $\Omega_{1,2}$ - постоянные величины.

Нахождение стационарных состояний с энергией $E$ сводится к подстановке в НУШ (3) волновой функции $\psi(x, t)=\psi(x) \exp (-i E t)$. В результате из (3) получается стационарное НУШ

$$
E \psi=-\frac{1}{2 m} \psi_{x x}^{\prime \prime}+\Omega(x) \psi-\gamma(x)|\psi|^{2} \psi+U(x) \psi
$$

Задача нахождения решения НУШ (4) с потенциалом (2) эквивалентна решению НУШ без потенциала

$$
\psi_{x x}^{\prime \prime}+2 m\left(E-\Omega(x)+\gamma(x) \psi^{2}\right) \psi=0,
$$

с двумя граничными условиями сопряжения в точке $x=0$, через которую проходит плоскость дефекта. Первое граничное условие соответствует требованию непрерывности волновой функции

$$
\psi(-0)=\psi(+0)=\psi(0)
$$

В $[17,18]$ для получения второго граничного условия проинтегрировали обе части уравнения (5) с потенциалом (2) по $x$ на малом интервале $[-\varepsilon ; \varepsilon]$ и устремили $\varepsilon$ к нулю. В результате получено второе граничное условие

$$
\psi^{\prime}(+0)-\psi^{\prime}(-0)=2 m U_{0} \psi^{3}(0) .
$$

В линейной среде без дефекта (всюду $\gamma(x) \equiv 0$ и $U(x) \equiv 0)$ распространяются свободные волны с квадратичным законом дисперсии $E=\Omega+k^{2} / 2 m$, где $k-$ 
волновое число. При наличии простого дефекта, описываемого короткодействующим потенциалом (1), в линейной среде существует симметричное локализованное по обе стороны от дефекта состояние. Такое состояние описывается волновой функцией $\psi(x)=A e^{-q|x|}$, где $q=-m U_{0}$, и существует только для притягивающего дефекта с $U_{0}<0$. Энергия такого локального уровня $E=\Omega-m U_{0}^{2} / 2$.

\section{2. Локализация возбуждений на границе между линейным кристаллом и самофокусирующей средой}

В случае контакта линейного кристалла с самофокусирующей средой, что отвечает положительной нелинейности $(\gamma>0)$ в уравнении $(5)$, в зависимости от энергии могут возникать два типа состояний с несимметричным относительно плоскости дефекта профилем поля. Первый тип описывается монотонно затухающими по обе стороны от дефекта решениями НУШ. Второй тип, называемый квазилокальным, состоит из локализованного в нелинейной среде состояния и стоячей волны в линейной среде.

\section{1. Локальные состояния}

В случае, когда энергия возбуждения лежит в диапазоне $E<\min \left\{\Omega_{1}, \Omega_{2}\right\}$, НУШ (5) имеет решение в виде

$$
\psi(x)= \begin{cases}\psi_{0 c} \exp \left(q_{1} x\right), & x<0, \\ A_{c} / \operatorname{ch} q_{2}\left(x-x_{0}\right), & x>0\end{cases}
$$

Параметры решения (8) определяются после его подстановки в уравнение (5) и условие непрерывности (6)

$$
\begin{gathered}
q_{1,2}^{2}=2 m\left(\Omega_{1,2}-E\right), \\
A_{c}^{2}=q_{2}^{2} /(m \gamma), \\
\psi_{0 c}=q_{2} /\left\{(m \gamma)^{1 / 2} \operatorname{ch} q_{2} x_{0}\right\} .
\end{gathered}
$$

Параметр $x_{0}$ характеризует положение „центра“ солитона в нелинейном кристалле справа от дефекта. Он связан с энергией локализации возбуждения, которая определяется из дисперсионного соотношения, получаемого после подстановки решения (8) в граничное условие (7)

$$
q_{2} \text { th } q_{2} x_{0}-q_{1}=2 U_{0} q_{2}^{2}\left(1-\operatorname{th}^{2} q_{2} x_{0}\right) / \gamma .
$$

Из соотношения (12) находится одно из волновых чисел (любое, $q_{1}$ или $q_{2}$, так как они связаны), которое позволяет определить энергию как функцию параметров $E=E\left(m, U_{0}, \gamma, x_{0}\right)$. Положение „центра“ солитона $x_{0}$ является свободным параметром. Анализ дисперсионного соотношения (12) проведем в различных частных случаях, допускающих нахождения его решения в явном виде.

В случае одинаковых значений $\Omega_{1}=\Omega_{2}=\Omega$ из (9) следует, что $q_{1}=q_{2}=q$. Тогда дисперсионное соотношение принимает вид (тривиальный случай, когда th $q x_{0}=1$, далее не рассматривается)

$$
\gamma=-2 U_{0} q\left(1+\text { th } q x_{0}\right) .
$$

Из (13) можно получить энергию в явном виде для локализованного состояния, положение „центра“ которого совпадает с плоскостью дефекта, т.е. $x_{0}=0$. В этом случае из (13) определяется декремент затухания: $q=-\gamma / 2 U_{0}$. Так как $q>0$ и в среде с самофокусировкой $\gamma>0$, должно быть $U_{0}<0$. Другими словами, локализованное состояние рассматриваемого вида существует только для притягивающего тонкого дефектного слоя с нелинейными свойствами, описываемого потенциалом (2), точно также как и для случая простого дефекта, описываемого потенциалом (1). Энергия локального состояния в таком случае определяется выражением

$$
E=\Omega-\gamma^{2} / 8 m U_{0}^{2} .
$$

Из (11) определяется амплитуда колебаний дефектного слоя

$$
\psi_{0 c}=-(\gamma / m)^{1 / 2} / 2 U_{0} .
$$

$\mathrm{B}$,длинноволновом“ п приближении при $q x_{0} \ll 1$ из (13) можно получить декремент затухания

$$
q=\left\{-1 \pm\left(1-2 \gamma x_{0} / U_{0}^{1 / 2}\right)\right\} / x_{0} .
$$

Знак в (16) выбирается в зависимости от знака $x_{0}$ и с учетом требования $q>0$.

Следует отметить, что условие „длинноволнового“ приближения $q x_{0} \ll 1$ означает близость энергии возбуждения к краю спектра, когда выполняется требование $|\Omega-E| \ll 1 / 2 m x_{0}^{2}$.

С учетом (16) и (9) можно получить энергию локализованного состояния в явном виде

$$
E=\Omega-\left\{-1 \pm\left(1-\gamma x_{0} / U_{0}\right)^{1 / 2}\right\}^{2} / 8 m x_{0}^{2} .
$$

Для существования такого локального состояния должно выполняться условие $x_{0}<U_{0} / 2 \gamma$.

Если теперь считать, что $\Omega_{1} \neq \Omega_{2}$, то для случая $x_{0}=0$ дисперсионное соотношение (12) примет вид

$$
q_{1}=-2 U_{0} q_{2}^{2} / \gamma
$$

Из (18) следует, что, так как $q_{1}>0$ и в среде с самофокусировкой $\gamma>0$, локализуются такие состояния вблизи притягивающего дефекта с $U_{0}<0$.

Из (18) с учетом (9) получается энергия локализованного состояния в явном виде

$$
E=\Omega_{2}-\Omega_{c 0}\left\{1 \pm\left[1+2\left(\Omega_{1}-\Omega_{2}\right) / \Omega_{c 0}\right]^{1 / 2}\right\},
$$

где $\Omega_{c 0}=\gamma^{2} / 16 m U_{0}^{2}$. Для существования такого локального состояния с энергией (19) должно выполняться условие $\Omega_{2}<\Omega_{1}+\gamma^{2} / 32 m U_{0}^{2}$. 
В „длинноволновом“ ${ }^{6}$ приближении при $q_{2} x_{0} \ll 1$ из (12) можно получить энергию локализованного состояния в явном виде

$$
E=\Omega_{2}-\Omega_{c x}\left\{1 \pm\left[1+2\left(\Omega_{1}-\Omega_{2}\right) / \Omega_{c x}\right]^{1 / 2}\right\},
$$

где $\Omega_{c x}=\gamma^{2} / 4 m\left(\gamma x_{0}-2 U_{0}\right)^{2}$.

\section{2. Квазилокальные состояния}

В случае, когда энергия возбуждения лежит в диапазоне $\Omega_{1}<E<\Omega_{2}$, НУШ (5) имеет решение в виде

$$
\psi(x)= \begin{cases}B_{c} \cos (k x+\varphi), & x<0 \\ A_{c} / \operatorname{ch} q_{2}\left(x-x_{0}\right), & x>0\end{cases}
$$

Для существования решения такого типа должно выполняться требование $\Omega_{1}<\Omega_{2}$. Параметры решения (21) определяются после его подстановки в уравнение (5) и условие непрерывности (6). Значение $q_{2}$ определяется выражениям (9), амплитуда $A_{c}$ определяется выражением (10), а характеристики волны в линейном кристалле имеют вид

$$
\begin{gathered}
k^{2}=2 m\left(E-\Omega_{1}\right), \\
B_{c}=\psi_{0 c} / \cos \varphi .
\end{gathered}
$$

После подстановки решения (21) в граничное условие (7) получается дисперсионное соотношение

$$
k \operatorname{tg} \varphi+q_{2} \operatorname{th} q_{2} x_{0}=2 U_{0} q_{2}^{2}\left(1-\operatorname{th}^{2} q_{2} x_{0}\right) / \gamma .
$$

Из соотношения (24) находится одно из волновых чисел (любое, $k$ или $q_{2}$, так как они связаны), которое позволяет определить энергию как функцию параметров $E=E\left(m, U_{0}, \gamma, \varphi, x_{0}\right)$. Теперь положение „центра“ солитона $x_{0}$ и фаза $\varphi$ являются свободными параметрами.

Решение (24) описывает состояние, при котором линейная волна после перехода через тонкий дефектный слой затухает в глубину ангармонического кристалла, т. е. происходит локализация волны. Поскольку энергия такого стационарного состояния находится в спектре линейных волн, а по одну сторону от плоскости дефекта возбуждение локализуется, состояния такого вида можно называть квазилокальными.

Если рассмотреть решение (21), для которого $x_{0}=0$, то из (24) определяется энергия такого квазилокального состояния

$$
E=\Omega_{2}-\Omega_{c 0}^{\varphi}\left\{-1 \pm\left[1-2\left(\Omega_{2}-\Omega_{1}\right) / \Omega_{c 0}^{\varphi}\right]^{1 / 2}\right\}
$$

где $\Omega_{c 0}^{\varphi}=\Omega_{c 0} \operatorname{tg}^{2} \varphi$. Рассматриваемое состояние и те, которые будут далее получены, существуют не при всех значениях фазы $\varphi$. Для существования такого локального состояния с энергией (25) должно выполняться условие для фазы $\operatorname{tg}^{2} \varphi>32 m U_{0}^{2}\left(\Omega_{2}-\Omega_{1}\right) / \gamma^{2}$.
В „длинноволновом“ приближении при $q_{2} x_{0} \ll 1$ из (24) можно получить энергию квазилокального состояния в явном виде

$$
E=\Omega_{2}-\Omega_{c x}^{\varphi}\left\{-1 \pm\left[1-2\left(\Omega_{2}-\Omega_{1}\right) / \Omega_{c x}^{\varphi}\right]^{1 / 2}\right\},
$$

где $\Omega_{c x}^{\varphi}=\Omega_{c x} \operatorname{tg}^{2} \varphi$. Для существования такого локального состояния с энергией (26) должно выполняться условие для фазы $\operatorname{tg}^{2} \varphi>8 m\left(\Omega_{2}-\Omega_{1}\right)\left(\gamma x_{0}-2 U_{0}\right)^{2} / \gamma^{2}$.

\section{3. Локализация возбуждений на границе между линейным кристаллом и дефокусирующей средой}

В случае контакта линейного кристалла с дефокусирующей средой, т.е. с кристаллом с отрицательной нелинейностью $(\gamma<0)$, в уравнении $(5)$ в зависимости от энергии могут возникать локальные и квазилокальные состояния. В отличие от контакта со средой с самофокусировкой, при контакте с дефокусирующей средой возникают два вида локальных состояний, а также два вида квазилокальных состояний, отличающихся профилем локализации амплитуды поля и диапазоном энергии, в котором они существуют.

При анализе стационарных состояний в дефокусирующей среде положим для удобства $g=-\gamma>0$.

\section{1. Локальные состояния}

Когда энергия возбуждения лежит в диапазоне $E<\min \left\{\Omega_{1}, \Omega_{2}\right\}$, НУШ (5) имеет решение вида

$$
\psi(x)= \begin{cases}\psi_{0 s} \exp \left(q_{1} x\right), & x<0 \\ A_{s} / \operatorname{sh} q_{2}\left(x-x_{0}\right), & x>0\end{cases}
$$

Для ограниченности решения (27) должно выполняться условие $x_{0}<0$. Параметры решения (27) определяются после его подстановки в уравнение (5) и условие непрерывности (6). Значения $q_{1,2}$ определяются выражениями (9), а амплитуды имеют вид

$$
\begin{gathered}
A_{s}^{2}=q_{2}^{2} /(m g), \\
\psi_{0 s}=-q_{2} /\left\{(m g)^{1 / 2} \operatorname{sh} q_{2} x_{0}\right\} .
\end{gathered}
$$

После подстановки решения (27) в граничное условие (7) получается дисперсионное соотношение

$$
q_{2} \operatorname{cth} q_{2} x_{0}-q_{1}=2 U_{0} q_{2}^{2}\left(\operatorname{cth}^{2} q_{2} x_{0}-1\right) / g .
$$

В „длинноволновом“ приближении при $q_{2} x_{0} \ll 1$ из (30) можно получить декремент затухания волны в линейной среде

$$
q_{1}\left(1-2 U_{0} / g x_{0}\right) / x_{0} .
$$

Из (31) следует, что, так как $q_{1}>0$ и $g>0$, локализуются такие состояния как вблизи притягивающего 
дефекта, так и отталкивающего при выполнении одного из условий: 1) $x_{0}>0$ и $U_{0}<g x_{0} / 2$ или 2) $x_{0}<0$ и $U_{0}>g x_{0} / 2$. Энергия такого состояния определяется после подстановки (31) в (9)

$$
E=\Omega_{1}-\left(1-2 U_{0} / g x_{0}\right)^{2} / 2 m x_{0}^{2} .
$$

Из (29) определяется амплитуда колебаний дефектного слоя в ,Длинноволновом“ приближении при $q_{2} x_{0} \ll 1$ : $\psi_{0 s}=-1 / x_{0}(m g)^{1 / 2}$.

В случае, когда энергия возбуждения лежит в диапазоне $\Omega_{2}<E<\Omega_{1}$, что возможно когда значения дна зоны сплошного спектра в нелинейной дефокусирующей среде ниже, чем в линейной $\left(\Omega_{2}<\Omega_{1}\right)$, НУШ (5) имеет другое решение:

$$
\psi(x)= \begin{cases}\psi_{0 t} \exp \left(q_{1} x\right), & x<0 \\ A_{t} \operatorname{th} q_{t}\left(x-x_{0}\right), & x>0\end{cases}
$$

Параметры решения (33) определяются после его подстановки в уравнение (5) и условие непрерывности (6). Значение $q_{1}$ определяется выражениям (9), а остальные характеристики имеют вид

$$
\begin{gathered}
q_{t}^{2}=m\left(E-\Omega_{1}\right), \\
A_{t}^{2}=q_{t}^{2} /(m g), \\
\psi_{0 t}=-q_{t} \text { th } q_{t} x_{0} /(m g)^{1 / 2} .
\end{gathered}
$$

После подстановки решения (33) в граничное условие (7) получается дисперсионное соотношение

$$
2 q_{t} / \operatorname{sh} 2 q_{t} x_{0}+q_{1}=-2 U_{0} q_{t}^{2} \operatorname{th}^{2} q_{t} x_{0} / g .
$$

В „длинноволновом“ приближении при $q_{t} x_{0} \ll 1$ из (37) получается энергия такого состояния

$$
E=\Omega_{2}+\Omega_{1 t}\left\{-1 \pm\left(1+\Omega_{2 t} / \Omega_{1 t}\right)^{1 / 2}\right\},
$$

где $\Omega_{1 t}=g / 4 m x_{0} U_{0}, \Omega_{2 t}=2\left(\Omega_{1}-\Omega_{2}\right)-1 / 2 m x_{0}^{2}$. Знак „+“ в (38) выбирается при $\Omega_{1 t}>0$, а знак ,-“ при $\Omega_{1 t}<0$.

Из (36) определяется амплитуда колебаний дефектного слоя в ,Длинноволновом “ приближении при $q_{t} x_{0} \ll 1: \psi_{0 t}=-q_{t}^{2} x_{0} /(m g)^{1 / 2}$.

\section{2. Квазилокальные состояния}

В случае контакта линейного кристалла с кристаллом с отрицательной нелинейностью $(\gamma=-g<0)$ в энергетическом диапазоне $\Omega_{1}<E<\Omega_{2}$ НУШ (5) имеет решение в виде

$$
\psi(x)= \begin{cases}B_{s} \cos (k x+\varphi), & x<0 \\ A_{s} / \operatorname{sh} q_{2}\left(x-x_{0}\right), & x>0 .\end{cases}
$$

Параметры решения (39) определяются после его подстановки в уравнение (5) и условие непрерывности (6). Значение $q_{2}$ определяется выражениям (9), амплитуда $A_{s}-(28)$, волновое число $k-(22)$, а амплитуда волны в линейном кристалле имеет вид

$$
B_{s}=\psi_{0 s} / \cos \varphi
$$

После подстановки решения (39) в граничное условие (7) получается дисперсионное соотношение

$$
k \operatorname{tg} \varphi+q_{2} \operatorname{cth} q_{2} x_{0}=2 U_{0} q_{2}^{2}\left(\operatorname{cth}^{2} q_{2} x_{0}-1\right) / g .
$$

Для такого квазилокального состояния положение „центра“ солитона $x_{0}$, которое должно быть отрицательным для ограниченности решения (39), и фаза $\varphi$ являются свободными параметрами.

В „длинноволновом“ приближении при $q_{2} x_{0} \ll 1$ из (41) можно получить энергию квазилокального состояния в явном виде

$$
E=\Omega_{1}+\left(1-2 U_{0} / g x_{0}\right)^{2} \operatorname{ctg}^{2} \varphi / 2 m x_{0}^{2} .
$$

В энергетическом диапазоне $E>\max \left\{\Omega_{1}, \Omega_{2}\right\}$ при контакте линейного кристалла с кристаллом с отрицательной нелинейностью НУШ (5) имеет другое решение

$$
\psi(x)= \begin{cases}B_{t} \cos (k x+\varphi), & x<0, \\ A_{t} \operatorname{th} q_{t}\left(x-x_{0}\right), & x>0 .\end{cases}
$$

Параметры решения (43) определяются после его подстановки в уравнение (5) и условие непрерывности (6). Значение $q_{t}$ определяется выражениям (34), амплитуда $A_{t}-(35)$, волновое число $k-(22)$, а амплитуда волны в линейном кристалле имеет вид

$$
B_{t}=\psi_{0 t} / \cos \varphi
$$

После подстановки решения (43) в граничное условие (7) получается дисперсионное соотношение

$$
k \operatorname{tg} \varphi-2 q_{t} / \operatorname{sh} 2 q_{t} x_{0}=2 U_{0} q_{t}^{2} \operatorname{th}^{2} q_{1} x_{0} / g .
$$

В ,длинноволновом“ приближении при $q_{t} x_{0} \ll 1$ из (45) получается энергия квазилокального состояния

$$
E=\Omega_{2}+\Omega_{1 t}^{\varphi}\left\{-1 \pm\left(1+\Omega_{2 t}^{\varphi} / \Omega_{1 t}^{\varphi}\right)^{1 / 2}\right\} .
$$

где $\Omega_{1 t}^{\varphi}=\Omega_{1 t} \operatorname{tg}^{2} \varphi, \Omega_{2 t}^{\varphi}=2\left(\Omega_{1}-\Omega_{2}\right)-\operatorname{ctg}^{2} \varphi / m x_{0}^{2}$. Знак „+ + в (46) выбирается при $\Omega_{1 t}^{\varphi}>0$, а знак „--“ при $\Omega_{1 t}^{\varphi}<0$. Для сушествования такого состояния должно выполняться условие $U_{0}<g \operatorname{tg}^{4} \varphi /\left\{8 x_{0}\left[m\left(\Omega_{2}-\right.\right.\right.$ $\left.\left.\left.-\Omega_{1}\right) \operatorname{tg}^{2} \varphi-1 / 2 x_{0}^{2}\right]\right\}$. Ясно, что его выполнение возможно как для притягивающего дефекта, так и отталкивающего.

\section{Заключение}

В работе показано, что вблизи тонкой прослойки с нелинейными свойствами, разделяющей линейную и нелинейную среды, могут существовать стационарные 
состояния нескольких типов. Такие состояния порождаются различными типами солитонных решений НУШ.

При математической формулировке модели для описания плоского дефекта с нелинейными свойствами применялся потенциал с квадратичным относительно искомого поля слагаемым, вид которого был предложен в $[17,18]$. Задача решения НУШ с таким потенциалом сводится к нахождению решения НУШ без потенциала с нелинейными граничными условиями. Найдены решения сформулированной контактной краевой задачи с такими условиями. Получены выражения для энергии в явной аналитической форме. Показано, что учет нелинейных свойств дефекта приводит к модификации профиля нелинейных локализованных возбуждений и области их существования.

Установлено, что на границе раздела с нелинейными свойствами между линейным и нелинейным кристаллами могут возникать два типа стационарных состояний. Один из них описывается профилем поля, монотонно затухающим, т.е. локализованным по обе стороны от плоскости дефекта, и поэтому называемым локализованным. Другой тип стационарных состояний описывается профилем поля, монотонно затухающим только по одну сторону от плоскости дефекта, в которой расположен нелинейный кристалл, а по другую сторону от плоскости дефекта, в которой расположен линейный кристалл, поле представляет собой стоячую линейную волну. Второй тип можно назвать квазилокальным состоянием.

Для каждого типа стационарных состояний найдены несколько видов распределений полей, существование которых определяется знаком нелинейности среды и диапазоном возможной энергии возбуждений.

В случае контакта линейного кристалла с самофокусирующей керровской средой, т.е. с кристаллом с положительной нелинейностью $(\gamma>0)$, когда энергия возбуждения лежит в диапазоне $E<\min \left\{\Omega_{1}, \Omega_{2}\right\}$, реализуется локальное состояние с несимметричным профилем, порождаемое солитонным решением НУШ с гиперболическим косинусом на полуоси в области нелинейного кристалла и экспоненциально затухающим решением на полуоси в области линейного кристалла. Также здесь возникает квазилокальное состояние с энергией в диапазоне $\Omega_{1}<E<\Omega_{2}$, т. е. когда уровень дна зоны сплошного спектра в нелинейном кристалле выше, чем в линейном. В области нелинейного кристалла профиль поля описывается солитонным решением НУШ с гиперболическим косинусом, а в области линейного кристалла - стоячей линейной волной.

В случае контакта линейного кристалла с дефокусирующей керровской средой, т.е. с кристаллом с отрицательной нелинейностью $(\gamma<0)$, имеются по два вида как локальных, так и квазилокальных состояний. Локализованные состояния с несимметричным профилем одного вида реализуются в диапазоне энергий возбуждений $E<\min \left\{\Omega_{1}, \Omega_{2}\right\}$. Они порождаются солитонным решением НУШ с гиперболическим синусом на полуоси в области нелинейного кристалла и экспоненциально затухающим решением на полуоси в области линейного кристалла.

Локализованные состояния с несимметричным профилем другого вида реализуются в диапазоне энергий возбуждений $\Omega_{2}<E<\Omega_{1}$, т. е. когда уровень дна зоны сплошного спектра в линейном кристалле выше, чем в нелинейном. Они порождаются солитонным решением НУШ с гиперболическим тангенсом на полуоси в области нелинейного кристалла и экспоненциально затухающим решением на полуоси в области линейного кристалла.

Также здесь возникают и два вида квазилокальных состояний. Один вид существует в диапазоне энергий возбуждений $\Omega_{1}<E<\Omega_{2}$, т. е. когда уровень дна зоны сплошного спектра в нелинейном кристалле выше, чем в линейном. Такой вид состояний в области нелинейного кристалла описывается солитонным решением НУШ с гиперболическим синусом, а в области линейного кристалла - стоячей линейной волной. Квазилокальное состояние другого вида реализуется в диапазоне $E>\max \left\{\Omega_{1}, \Omega_{2}\right\}$. Профиль поля в нем в области нелинейного кристалла описывается солитонным решением НУШ с гиперболическим тангенсом, а в области линейного кристалла - стоячей линейной волной.

Таким образом, управляя энергией возбуждения, можно получать различные профили локализации поля по разные стороны от плоскости дефекта.

Рассмотрение модели тонкого дефектного слоя с нелинейными свойствами, описываемого потенциалом (2), привносит новые особенности в строение спектра локализованных состояний, в отличие от модели простого дефекта, описываемого потенциалом (1). Основное различие заключается в дисперсионных уравнения, и, как следствие, в уровнях энергии и областях существования состояний.

Результаты, полученные в работе, могут служить продолжением исследований особенностей локализации нелинейных возбуждений в средах с нелинейными дефектами на случай границы раздела линейной и нелинейной сред, проведенных в [16-18].

\section{Список литературы}

[1] Каплан А.Е. // Письма в ЖЭТФ. 1976. Т. 24. № 3. C. $132-137$.

[2] Павло В.И., Солодов И.Ю. // ФТТ. 1977. Т. 19. № 10. C. 2948-2954.

[3] Наянов В.И. // Письма в ЖЭТФ. 1986. Т. 44. № 5. C. 245-249.

[4] Паняев И.С., Санников Д.Г. // Компьютерная оптика. 2017. T. 41. № 2. C. 183-191.

[5] Ахмедиев Н.Н., Корнеев В.И., Кузьменко Ю.В. // ЖЭТФ. 1985. T. 88. № 1. C. 107-115.

[6] Shadrivov I.V., Sukhorukov A.A., Kivshar Yu.S., Zharov A.A., Boardman A.D., Egan P. // Phys. Rev. E. 2004. Vol. 69. P. 016617-1-9. 
[7] Михалаке Д., Назмитдинов Р.Г., Федянин В.К. // Физика элементарных частиц и атомного ядра. 1989. Т. 20. № 1. C. $198-253$.

[8] Горенцвейг В.И., Кившарь Ю.С., Косевич А.М., Сыркин Е.C. // ФНТ. 1990. Т. 16. № 11. С. 1472-1482.

[9] Abdullaev F.Kh., Baizakov B.B., Umarov B.A. // Optics Communications. 1998. Vol. 156. P. 341-346.

[10] Савотченко С.E. // Известия вузов. Физика. 2004. Т. 47. № 5. C. 79-84.

[11] Савотченко C.E. // Конденсированные среды и межфазные границы. 2017. № 2. С. 291-295.

[12] Савотченко C.E. // Вестник Воронежского гос. ун-та. Серия: Физика. Математика. 2016. № 4. С. 51-59.

[13] Kivshar U.S., Kosevich A.M., Chubykalo O.A. // Phys. Rev. A. 1990. Vol. 41. N. 3. P. $1677-1688$.

[14] Герасимчук И.В., Горобеи, Ю.И., Герасимчук В.С. // Journal of nano- and electronic physics. 2016. Vol. 8. N 2. P. 02020-1-7.

[15] Богдан М.М., Герасимчук И.В., Ковалев А.С. // ФНТ. 1997. T. 23. № 2. C. 197-207.

[16] Gerasimchuk I.V. // Journal of nano- and electronic physics. 2012. Vol. 4. N 4. P. 04024-1-4.

[17] Gerasimchuk I.V., Gorbach P.K., Dovhopolyi P.P. // Ukr. J. Phys. 2012. Vol. 57. N 6. P. 678-683.

[18] Герасимчук И.В. // ЖЭТФ. 2015. Т. 121. № 4. С. 596-605. 